\title{
A New Conservative Difference Scheme for the General Rosenau-RLW Equation
}

\author{
Jin-Ming Zuo, ${ }^{1}$ Yao-Ming Zhang, ${ }^{1}$ Tian-De Zhang, ${ }^{2}$ \\ and Feng Chang ${ }^{2}$ \\ ${ }^{1}$ School of Science, Shandong University of Technology, Zibo 255049, China \\ 2 School of Mathematics, Shandong University, Jinan 250100, China \\ Correspondence should be addressed to Jin-Ming Zuo, zuojinming@sdut.edu.cn
}

Received 28 May 2010; Accepted 14 October 2010

Academic Editor: Colin Rogers

Copyright (c) 2010 Jin-Ming Zuo et al. This is an open access article distributed under the Creative Commons Attribution License, which permits unrestricted use, distribution, and reproduction in any medium, provided the original work is properly cited.

A new conservative finite difference scheme is presented for an initial-boundary value problem of the general Rosenau-RLW equation. Existence of its difference solutions are proved by Brouwer fixed point theorem. It is proved by the discrete energy method that the scheme is uniquely solvable, unconditionally stable, and second-order convergent. Numerical examples show the efficiency of the scheme.

\section{Introduction}

In this paper, we consider the following initial-boundary value problem of the general Rosenau-RLW equation:

$$
u_{t}-u_{x x t}+u_{x x x x t}+u_{x}+\left(u^{p}\right)_{x}=0 \quad\left(x_{l}<x<x_{r}, 0<t<T\right)
$$

with an initial condition

$$
u(x, 0)=u_{0}(x) \quad\left(x_{l} \leq x \leq x_{r}\right)
$$

and boundary conditions

$$
u\left(x_{l}, t\right)=u\left(x_{r}, t\right)=0, \quad u_{x x}\left(x_{l}, t\right)=u_{x x}\left(x_{r}, t\right)=0 \quad(0 \leq t \leq T),
$$


where $p \geq 2$ is a integer and $u_{0}(x)$ is a known smooth function. When $p=2$, (1.1) is called as usual Rosenau-RLW equation. When $p=3$, (1.1) is called as modified Rosenau-RLW (MRosenau-RLW) equation. The initial boundary value problem (1.1)-(1.3) possesses the following conservative quantities:

$$
\begin{gathered}
Q(t)=\frac{1}{2} \int_{x_{l}}^{x_{r}} u(x, t) d x=\frac{1}{2} \int_{x_{l}}^{x_{r}} u_{0}(x, t) d x=Q(0), \\
E(t)=\frac{1}{2}\left(\|u\|_{L^{2}}^{2}+\left\|u_{x}\right\|_{L^{2}}^{2}+\left\|u_{x x}\right\|_{L^{2}}^{2}\right)=\frac{1}{2}\left(\left\|u_{0}\right\|_{L^{2}}^{2}+\left\|u_{0 x}\right\|_{L^{2}}^{2}+\left\|u_{0 x x}\right\|_{L^{2}}^{2}\right)=E(0) .
\end{gathered}
$$

It is known the conservative scheme is better than the nonconservative ones. Zhang et al. [1] point out that the nonconservative scheme may easily show nonlinear blow up. In [2] $\mathrm{Li}$ and $\mathrm{Vu}$-Quoc said "... in some areas, the ability to preserve some invariant properties of the original differential equation is a criterion to judge the success of a numerical simulation". In [3-11], some conservative finite difference schemes were used for a system of the generalized nonlinear Schrödinger equations, Regularized long wave (RLW) equations, Sine-Gordon equation, Klein-Gordon equation, Zakharov equations, Rosenau equation, respectively. Numerical results of all the schemes are very good. Hence, we propose a new conservative difference scheme for the general Rosenau-RLW equation, which simulates conservative laws (1.4) and (1.5) at the same time. The outline of the paper is as follows. In Section 2, a nonlinear difference scheme is proposed and corresponding convergence and stability of the scheme are proved. In Section 3, some numerical experiments are shown.

\section{A Nonlinear-Implicit Conservative Scheme}

In this section, we propose a nonlinear-implicit conservative scheme for the initial-boundary value problem (1.1)-(1.3) and give its numerical analysis.

\subsection{The Nonlinear-Implicit Scheme and Its Conservative Law}

For convenience, we introduce the following notations

$$
x_{j}=x_{r}+j h, \quad t_{n}=n \tau, \quad j=0,1, \ldots, J, \quad n=0,1, \ldots, \quad\left[\frac{T}{\tau}\right]=N,
$$

where $h=\left(x_{r}-x_{l}\right) / J$ and $\tau$ denote the spatial and temporal mesh sizes, $u_{j}^{n} \equiv u\left(x_{j}, t_{n}\right)$, $U_{j}^{n} \approx u\left(x_{j}, t_{n}\right)$, respectively,

$$
\begin{gathered}
\left(U_{j}^{n}\right)_{t}=\frac{U_{j}^{n+1}-U_{j}^{n}}{\tau}, \quad\left(U_{j}^{n}\right)_{x}=\frac{U_{j+1}^{n}-U_{j}^{n}}{h}, \quad\left(U_{j}^{n}\right)_{\bar{x}}=\frac{U_{j}^{n}-U_{j-1}^{n}}{h}, \\
\left(U_{j}^{n}\right)_{\hat{x}}=\frac{1}{2}\left(\left(U_{j}^{n}\right)_{x}+\left(U_{j}^{n}\right)_{\bar{x}}\right), \quad U_{j}^{n+1 / 2}=\frac{1}{2}\left(U_{j}^{n+1}+U_{j}^{n}\right), \quad\left\langle U^{n}, V^{n}\right\rangle=h \sum_{j=1}^{J-1} U_{j}^{n} V_{j}^{n}, \\
\left\|U^{n}\right\|^{2}=\left\langle U^{n}, U^{n}\right\rangle, \quad\left\|U^{n}\right\|_{\infty}=\max _{1 \leq j \leq J}\left|U_{j}^{n}\right|,
\end{gathered}
$$


and in the paper, $C$ denotes a general positive constant, which may have different values in different occurrences.

Since $\left(u^{p}\right)_{x}=(2 /(p+1)) \sum_{i=0}^{p-1} u^{i}\left(u^{p-i}\right)_{x}$, then the finite difference scheme for the problem (1.1)-(1.3) is written as follows:

$$
\begin{gathered}
\left(U_{j}^{n}\right)_{t}-\left(U_{j}^{n}\right)_{x \bar{x} t}+\left(U_{j}^{n}\right)_{x x \bar{x} \bar{x} t}+\left(U_{j}^{n+1 / 2}\right)_{\hat{x}}+\frac{2}{p+1} \sum_{i=0}^{p-1}\left(U_{j}^{n+1 / 2}\right)^{i}\left[\left(U_{j}^{n+1 / 2}\right)^{p-i}\right]_{\widehat{x}}=0 \\
j=1,2, \ldots, J-1 ; \quad n=1,2, \ldots, N, \\
U_{j}^{0}=u_{0}\left(x_{j}\right), \quad j=0,1,2, \ldots, J \\
U_{0}^{n}=U_{J}^{n}=0, \quad\left(U_{0}^{n}\right)_{x \bar{x}}=\left(U_{J}^{n}\right)_{x \bar{x}}=0, \quad n=1,2, \ldots, N .
\end{gathered}
$$

Lemma 2.1 (see [12]). For any two mesh functions, $U, V \in Z_{h^{\prime}}^{0}$ one has

$$
\begin{gathered}
\left\langle(U)_{x}, V\right\rangle=-\left\langle U,(V)_{\bar{x}}\right\rangle, \\
\left\langle(U)_{\widehat{x}}, V\right\rangle=-\left\langle U,(V)_{\widehat{x}}\right\rangle, \\
\left\langle V,(U)_{x \bar{x}}\right\rangle=-\left\langle(V)_{x},(U)_{x}\right\rangle, \\
\left\langle U,(U)_{x \bar{x}}\right\rangle=-\left\langle(U)_{x},(U)_{x}\right\rangle=-\left\|U_{x}\right\|^{2} .
\end{gathered}
$$

Furthermore, if $\left(U_{0}^{n}\right)_{x \bar{x}}=\left(U_{J}^{n}\right)_{x \bar{x}}=0$, then

$$
\left\langle U,(U)_{x x \overline{x x}}\right\rangle=\left\|U_{x x}\right\|^{2} .
$$

Theorem 2.2. Suppose that $u_{0} \in H_{0}^{2}\left[x_{l}, x_{r}\right]$, then scheme (2.3)-(2.5) is conservative in the senses:

$$
\begin{gathered}
Q^{n}=\frac{h}{2} \sum_{j=1}^{J-1} U_{j}^{n}=Q^{n-1}=\cdots=Q^{0}, \\
E^{n}=\frac{1}{2}\left\|U^{n}\right\|^{2}+\frac{1}{2}\left\|U_{x}^{n}\right\|^{2}+\frac{1}{2}\left\|U_{x x}^{n}\right\|^{2}=E^{n-1}=\cdots=E^{0} .
\end{gathered}
$$

Proof. Multiplying (2.3) with $h / 2$, according to boundary condition (2.5), and then summing up for $j$ from 1 to $J-1$, we have

$$
\frac{h}{2} \sum_{j=1}^{J-1}\left(U_{j}^{n+1}-U_{j}^{n}\right)=0 .
$$


Let

$$
Q^{n}=\frac{h}{2} \sum_{j=1}^{J-1} U_{j}^{n}
$$

Then (2.8) is gotten from (2.10).

Computing the inner product of (2.3) with $U^{n+1 / 2}$, according to boundary condition (2.5) and Lemma 2.1, we obtain

$$
\frac{1}{2}\left\|U^{n}\right\|_{t}^{2}+\frac{1}{2}\left\|U_{x}^{n}\right\|_{t}^{2}+\frac{1}{2}\left\|U_{x x}^{n}\right\|_{t}^{2}+\left\langle\left(U^{n+1 / 2}\right)_{\widehat{x}^{\prime}} U^{n+1 / 2}\right\rangle+\left\langle\kappa\left(U^{n+1 / 2}, U^{n+1 / 2}\right), U^{n+1 / 2}\right\rangle=0
$$

where

$$
\begin{gathered}
\mathcal{\kappa}\left(U^{n+1 / 2}, U^{n+1 / 2}\right)=\frac{2}{p+1} \sum_{i=0}^{p-1}\left(U^{n+1 / 2}\right)^{i}\left[\left(U^{n+1 / 2}\right)^{p-i}\right]_{\widehat{x}}, \\
U^{n+1 / 2}=\frac{1}{2}\left(U^{n+1}+U^{n}\right) .
\end{gathered}
$$

According to

$$
\begin{gathered}
\left\langle\left(U^{n+1 / 2}\right)_{\widehat{x}^{\prime}} U^{n+1 / 2}\right\rangle=0, \\
\left\langle\kappa\left(U^{n+1 / 2}, U^{n+1 / 2}\right), U^{n+1 / 2}\right\rangle=\frac{2}{p+1}\left\langle\sum_{i=0}^{p-1}\left(U^{n+1 / 2}\right)^{i}\left[\left(U^{n+1 / 2}\right)^{p-i}\right]_{\widehat{x}^{\prime}} U^{n+1 / 2}\right\rangle \\
=-\frac{2}{p+1}\left\langle\sum_{i=0}^{p-1}\left[\left(U^{n+1 / 2}\right)^{i+1}\right]_{\widehat{x}^{\prime}}\left(U^{n+1 / 2}\right)^{p-i}\right\rangle \\
=-\frac{2}{p+1}\left\langle\sum_{i=0}^{p-1}\left(U^{n+1 / 2}\right)^{i}\left[\left(U^{n+1 / 2}\right)^{p-i}\right]_{\widehat{x}^{\prime}} U^{n+1 / 2}\right\rangle,
\end{gathered}
$$

we have $\left\langle\kappa\left(U^{n+1 / 2}, U^{n+1 / 2}\right), U^{n+1 / 2}\right\rangle=0$. It follows from (2.12) that

$$
\frac{1}{2}\left\|U^{n}\right\|_{t}^{2}+\frac{1}{2}\left\|U_{x}^{n}\right\|_{t}^{2}+\frac{1}{2}\left\|U_{x x}^{n}\right\|_{t}^{2}=0 .
$$

Let

$$
E^{n}=\frac{1}{2}\left\|U^{n}\right\|^{2}+\frac{1}{2}\left\|U_{x}^{n}\right\|^{2}+\frac{1}{2}\left\|U_{x x}^{n}\right\|^{2} .
$$

Then (2.9) is gotten from (2.15). This completes the proof of Theorem 2.2. 


\subsection{Existence and Prior Estimates of Difference Solution}

To show the existence of the approximations $U^{n}(n=1,2, \ldots, N)$ for scheme (2.3)-(2.5), we introduce the following Brouwer fixed point theorem [13].

Lemma 2.3. Let $H$ be a finite-dimensional inner product space, $\|\cdot\|$ be the associated norm, and $g: H \rightarrow H$ be continuous. Assume, moreover, that there exist $\alpha>0$, for all $z \in H,\|z\|=\alpha$, $\langle\omega(z), z\rangle>0$. Then, there exists $a z^{*} \in H$ such that $g\left(z^{*}\right)=0$ and $\left\|z^{*}\right\| \leq \alpha$.

following.

Let $Z_{h}^{0}=\left\{v=\left(v_{j}\right) \mid v_{0}=v_{J}=\left(v_{0}\right)_{x \bar{x}}=\left(v_{J}\right)_{x \bar{x}}=0, j=0,1, \ldots, J\right\}$, then have the

Theorem 2.4. There exists $U^{n+1} \in Z_{h}^{0}$ which satisfies scheme (2.3)-(2.5).

Proof (by Brouwer fixed point theorem). It follows from the original problem (1.1)-(1.3) that $U^{0}$ satisfies scheme (2.3)-(2.5). Assume there exists $U^{1}, U^{2}, \ldots, U^{n} \in Z_{h}^{0}$ which satisfy scheme (2.3)-(2.5), as $n \leq N-1$, now we try to prove that $U^{n+1} \in Z_{h}^{0}$, satisfy scheme (2.3)-(2.5).

We define $\omega$ on $Z_{h}^{0}$ as follows:

$$
\omega(v)=2 v-2 U^{n}-2 v_{x \bar{x}}+2 U_{x \bar{x}}+2 v_{x x \overline{x x}}-2 U_{x x \overline{x x}}+\tau v_{\hat{x}}+\tau \kappa(v, v),
$$

where $\kappa(v, v)=(2 /(p+1)) \sum_{i=0}^{p-1} v^{i}\left(v^{p-i}\right)_{\hat{x}}$. Computing the inner product of (2.17) with $v$ and considering $\langle\kappa(v, v), v\rangle=0$ and $\left\langle v_{\hat{x}}, v\right\rangle=0$, we obtain

$$
\begin{aligned}
\langle\omega(v), v\rangle= & 2\|v\|^{2}+2\left\|v_{x}\right\|^{2}+2\left\|v_{x x}\right\|^{2}-2\left\langle U^{n}, v\right\rangle+2\left\langle U_{x \bar{x}}^{n}, v\right\rangle-2\left\langle U_{x x \overline{x x}}^{n}, v\right\rangle \\
\geq & 2\|v\|^{2}+2\left\|v_{x}\right\|^{2}+2\left\|v_{x x}\right\|^{2}-\left(\left\|U^{n}\right\|^{2}+\|v\|^{2}\right) \\
& -\left(\left\|U_{x}^{n}\right\|^{2}+\left\|v_{x}\right\|^{2}\right)-\left(\left\|U_{x x}^{n}\right\|^{2}+\left\|v_{x x}\right\|^{2}\right) \\
= & \|v\|^{2}+\left\|v_{x}\right\|^{2}+\left\|v_{x x}\right\|^{2}-\left(\left\|U^{n}\right\|^{2}+\left\|U_{x}^{n}\right\|^{2}+\left\|U_{x x}^{n}\right\|^{2}\right) \\
\geq & \|v\|^{2}-\left(\left\|U^{n}\right\|^{2}+\left\|U_{x}^{n}\right\|^{2}+\left\|U_{x x}^{n}\right\|^{2}\right) .
\end{aligned}
$$

Hence, for all $v \in Z_{h^{\prime}}^{0}\|v\|^{2}=\left\|U^{n}\right\|^{2}+\left\|U_{x}^{n}\right\|^{2}+\left\|U_{x x}^{n}\right\|^{2}+1$ there exists $\langle\omega(v), v\rangle \geq 0$. It follows from Lemma 2.3 that exists $v^{*} \in Z_{h}^{0}$ which satisfies $\omega\left(v^{*}\right)=0$. Let $U^{n+1}=2 v-U^{n}$, then it can be proved that $U^{n+1} \in Z_{h}^{0}$ is the solution of scheme (2.3)-(2.5). This completes the proof of Theorem 2.4.

Next we will give some priori estimates of difference solutions. First the following two lemmas [14] are introduced:

Lemma 2.5 (discrete Sobolev's estimate). For any discrete function $\left\{u_{j}^{n} \mid j=0,1, \ldots, J\right\}$ on the finite interval $\left\{x_{l}, x_{r}\right\}$, there is the inequality

$$
\left\|u^{n}\right\|_{\infty} \leq \varepsilon\left\|u_{x}^{n}\right\|+C(\varepsilon)\left\|u^{n}\right\|,
$$

where $\varepsilon, C(\varepsilon)$ are two constants independent of $\left\{u_{j}^{n} \mid j=0,1, \ldots, J\right\}$ and step length $h$. 
Lemma 2.6 (discrete Gronwall's inequality). Suppose that the discrete function $\left\{w^{n} \mid n=0,1\right.$, $\ldots, N\}$ satisfies the inequality

$$
w^{n}-w^{n-1} \leq A \tau w^{n}+B \tau w^{n-1}+C_{n} \tau
$$

where $A, B$ and $C_{n}(n=0,1,2, \ldots, N)$ are nonnegative constants. Then

$$
\max _{1 \leq n \leq N}\left|w^{n}\right| \leq\left(w^{0}+\tau \sum_{l=1}^{N} C_{l}\right) e^{2(A+B) T}
$$

where $\tau$ is sufficiently small, such that $(A+B) \tau \leq(N-1) / 2 N,(N>1)$.

Theorem 2.7. Suppose that $u_{0} \in H_{0}^{2}\left[x_{l}, x_{r}\right]$, then the following inequalities

$$
\left\|U^{n}\right\| \leq C, \quad\left\|U_{x}^{n}\right\| \leq C, \quad\left\|U^{n}\right\|_{\infty} \leq C, \quad\left\|U_{x x}^{n}\right\| \leq C .
$$

hold.

Proof. It is follows from (2.9) that

$$
\left\|U^{n}\right\| \leq C, \quad\left\|U_{x}^{n}\right\| \leq C, \quad\left\|U_{x x}^{n}\right\| \leq C .
$$

According to Lemma 2.5, we obtain

$$
\left\|U^{n}\right\|_{\infty} \leq C
$$

This completes the proof of Theorem 2.7.

Remark 2.8. Theorem 2.7 implies that scheme (2.3)-(2.5) is unconditionally stable.

\subsection{Convergence and Uniqueness of Difference Solution}

First, we consider the convergence of scheme (2.3)-(2.5). We define the truncation error as follows:

$$
\begin{array}{r}
r_{j}^{n}=\left(u_{j}^{n}\right)_{t}-\left(u_{j}^{n}\right)_{x \bar{x} t}+\left(u_{j}^{n}\right)_{x x \overline{x x} t}+\left(u_{j}^{n+1 / 2}\right)_{\widehat{x}}+\frac{2}{p+1} \sum_{i=0}^{p-1}\left(u_{j}^{n+1 / 2}\right)^{i}\left[\left(u_{j}^{n+1 / 2}\right)^{p-i}\right]_{\widehat{x}} \\
j=1,2, \ldots, J-1 ; \quad n=1,2, \ldots, N,
\end{array}
$$

then from Taylor's expansion, we obtain the following. 
Theorem 2.9. Suppose that $u_{0} \in H_{0}^{2}\left[x_{l}, x_{r}\right]$ and $u(x, t) \in C^{5,3}$, then the truncation errors of scheme (2.3)-(2.5) satisfy

$$
\left|r_{j}^{n}\right|=O\left(\tau^{2}+h^{2}\right)
$$

as $\tau \rightarrow 0, h \rightarrow 0$.

Theorem 2.10. Suppose that the conditions of Theorem 2.9 are satisfied, then the solution of scheme (2.3)-(2.5) converges to the solution of problem (1.1)-(1.3) with order $O\left(\tau^{2}+h^{2}\right)$ in the $L_{\infty}$ norm.

Proof. Subtracting (2.3) from (2.25) letting

$$
e_{j}^{n}=u_{j}^{n}-U_{j}^{n}
$$

we obtain

$$
r_{j}^{n}=\left(e_{j}^{n}\right)_{t}-\left(e_{j}^{n}\right)_{x \bar{x} t}+\left(e_{j}^{n}\right)_{x x \overline{x x} t}+\left(e_{j}^{n+1 / 2}\right)_{\widehat{x}}+\kappa\left(u_{j}^{n+1 / 2}, u_{j}^{n+1 / 2}\right)-\kappa\left(U_{j}^{n+1 / 2}, U_{j}^{n+1 / 2}\right) .
$$

Computing the inner product of (2.28) with $2 e^{n+1 / 2}$, we obtain

$$
\begin{aligned}
\left\langle 2 r^{n}, e^{n+1 / 2}\right\rangle= & \left\|e^{n}\right\|_{t}^{2}+\left\|e_{x}^{n}\right\|_{t}^{2}+\left\|e_{x x}^{n}\right\|_{t}^{2}+2\left\langle\left(e_{j}^{n+1 / 2}\right)_{\widehat{x}^{\prime}} e_{j}^{n+1 / 2}\right\rangle \\
& +2\left\langle\kappa\left(u_{j}^{n+1 / 2}, u_{j}^{n+1 / 2}\right)-\kappa\left(U_{j}^{n+1 / 2}, U_{j}^{n+1 / 2}\right), e^{n+1 / 2}\right\rangle .
\end{aligned}
$$

From the conservative property (1.5), it can be proved by Lemma 2.5 that $\|u\|_{L_{\infty}} \leq C$. Then by Theorem 2.7 we can estimate (2.29) as follows:

$$
\begin{aligned}
& \left\langle\kappa\left(u_{j}^{n+1 / 2}, u_{j}^{n+1 / 2}\right)-\kappa\left(U_{j}^{n+1 / 2}, U_{j}^{n+1 / 2}\right), e^{n+1 / 2}\right\rangle \\
& =\frac{2}{p+1} h \sum_{j=1}^{J-1}\left\{\sum_{i=0}^{p-1}\left(u_{j}^{n+1 / 2}\right)^{i}\left[\left(u_{j}^{n+1 / 2}\right)^{p-i}\right]_{\widehat{x}}-\sum_{i=0}^{p-1}\left(U_{j}^{n+1 / 2}\right)^{i}\left[\left(u_{j}^{n+1 / 2}\right)^{p-i}\right]_{\widehat{x}}\right\}\left(e_{j}^{n+1 / 2}\right) \\
& =\frac{2}{p+1} h \sum_{j=1}^{J-1}\left\{\sum_{i=0}^{p-1}\left(e_{j}^{n+1 / 2}\right)^{i} \sum_{r=0}^{i-1}\left[\left(u_{j}^{n+1 / 2}\right)^{i-1-r}\left(U_{j}^{n+1 / 2}\right)^{r}\right]\left[\left(u_{j}^{n+1 / 2}\right)^{p-i}\right]_{\widehat{x}}\right. \\
& \left.\quad-\sum_{i=0}^{p-1}\left(U_{i}^{n+1 / 2}\right)^{i}\left[\left(e_{j}^{n+1 / 2}\right)^{p-i-1} \sum_{r=0}\left[\left(u_{j}^{n+1 / 2}\right)^{p-i-1-r}\left(U_{j}^{n+1 / 2}\right)^{r}\right]\right]\right\}\left(e_{j}^{n+1 / 2}\right) \\
& \leq C\left(\left\|e^{n}\right\|^{2}+\left\|e^{n+1}\right\|^{2}+\left\|e_{x}^{n}\right\|^{2}+\left\|e_{x}^{n+1}\right\|^{2}\right) .
\end{aligned}
$$


According to the following inequality [11]

$$
\begin{gathered}
\left\|e_{x}^{n}\right\|^{2} \leq \frac{1}{2}\left(\left\|e^{n}\right\|^{2}+\left\|e_{x x}^{n}\right\|^{2}\right), \quad\left\|e_{x}^{n+1}\right\|^{2} \leq \frac{1}{2}\left(\left\|e^{n+1}\right\|^{2}+\left\|e_{x x}^{n+1}\right\|^{2}\right), \\
\left\langle\left(e_{j}^{n+1 / 2}\right)_{\widehat{x}^{\prime}} e_{j}^{n+1 / 2}\right\rangle=0 \\
\left\langle 2 r^{n}, e^{n+1 / 2}\right\rangle \leq\left\|r^{n}\right\|^{2}+\left(\left\|e^{n}\right\|^{2}+\left\|e^{n+1}\right\|^{2}\right) .
\end{gathered}
$$

Substituting (2.30)-(2.31) into (2.29), we obtain

$$
\left\|e^{n}\right\|_{t}^{2}+\left\|e_{x}^{n}\right\|_{t}^{2}+\left\|e_{x x}^{n}\right\|_{t}^{2} \leq\left\|r^{n}\right\|^{2}+C\left(\left\|e^{n}\right\|^{2}+\left\|e^{n+1}\right\|^{2}+\left\|e_{x}^{n}\right\|^{2}+\left\|e_{x}^{n+1}\right\|^{2}+\left\|e_{x x}^{n}\right\|^{2}+\left\|e_{x x}^{n+1}\right\|^{2}\right) .
$$

Let

$$
B^{n}=\left\|e^{n}\right\|^{2}+\left\|e_{x}^{n}\right\|^{2}+\left\|e_{x x}^{n}\right\|^{2},
$$

then (2.32) can be rewritten as

$$
B^{n}-B^{n-1} \leq C \tau\left(\tau^{2}+h^{2}\right)^{2}+C \tau\left(B^{n}-B^{n-1}\right) .
$$

Choosing suitable $\tau$ which is small enough, we obtain by Lemma 2.6 that

$$
B^{n} \leq C\left(B^{0}+\left(\tau^{2}+h^{2}\right)^{2}\right)
$$

From the discrete initial conditions, we know that $e^{0}$ is of second-order accuracy, then

$$
B^{0}=O\left(\tau^{2}+h^{2}\right)^{2}
$$

Then we have

$$
\left\|e^{n}\right\| \leq O\left(\tau^{2}+h^{2}\right), \quad\left\|e_{x}^{n}\right\| \leq O\left(\tau^{2}+h^{2}\right), \quad\left\|e_{x x}^{n}\right\| \leq O\left(\tau^{2}+h^{2}\right)
$$

It follows from Lemma 2.5, we have $\left\|e^{n}\right\|_{\infty} \leq O\left(\tau^{2}+h^{2}\right)$. This completes the proof of Theorem 2.10. 
Table 1: The errors of numerical solutions at $t=60$ with $\tau=h$ for $p=2$.

\begin{tabular}{lcccc}
\hline$h$ & $\left\|u^{n}-U^{n}\right\|$ & $\left\|u^{n}-U^{n}\right\|_{\infty}$ & $\left\|u^{n / 4}-U^{n / 4}\right\| /\left\|u^{n}-U^{n}\right\|$ & $\left\|u^{n / 4}-U^{n / 4}\right\|_{\infty} /\left\|u^{n}-U^{n}\right\|_{\infty}$ \\
\hline 0.4 & $5.476327 \times 10^{-2}$ & $1.958718 \times 10^{-2}$ & & \\
0.2 & $1.385256 \times 10^{-2}$ & $4.983761 \times 10^{-3}$ & 3.953296 & 3.930200 \\
0.1 & $3.474318 \times 10^{-3}$ & $1.252185 \times 10^{-3}$ & 3.987130 & 3.980050 \\
0.05 & $8.691419 \times 10^{-4}$ & $3.134571 \times 10^{-4}$ & 3.997412 & 3.994759 \\
0.025 & $2.059064 \times 10^{-4}$ & $7.550730 \times 10^{-5}$ & 4.221051 & 4.151348 \\
\hline
\end{tabular}

Table 2: The errors of numerical solutions at $t=60$ with $\tau=h$ for $p=3$.

\begin{tabular}{lcccc}
\hline$h$ & $\left\|u^{n}-U^{n}\right\|$ & $\left\|u^{n}-U^{n}\right\|_{\infty}$ & $\left\|u^{n / 4}-U^{n / 4}\right\| /\left\|u^{n}-U^{n}\right\|$ & $\left\|u^{n / 4}-U^{n / 4}\right\|_{\infty} /\left\|u^{n}-U^{n}\right\|_{\infty}$ \\
\hline 0.4 & $1.164674 \times 10^{-1}$ & $4.251029 \times 10^{-2}$ & & \\
0.2 & $2.940136 \times 10^{-2}$ & $1.080424 \times 10^{-2}$ & 3.961294 & 3.934592 \\
0.1 & $7.357052 \times 10^{-3}$ & $2.708996 \times 10^{-3}$ & 3.996350 & 3.988283 \\
0.05 & $1.837759 \times 10^{-3}$ & $6.772212 \times 10^{-4}$ & 4.003273 & 4.000165 \\
0.025 & $4.283535 \times 10^{-4}$ & $1.596208 \times 10^{-4}$ & 4.290286 & 4.242688 \\
\hline
\end{tabular}

Theorem 2.11. Scheme (2.3)-(2.5) is uniquely solvable.

Proof. Assume that $U^{n}$ and $U^{\prime n}$ both satisfy scheme (2.3)-(2.5), let $W^{n}=U^{n}-U^{\prime n}$, we obtain

$$
\begin{gathered}
\left(W_{j}^{n}\right)_{t}-\left(W_{j}^{n}\right)_{x \bar{x} t}+\left(W_{j}^{n}\right)_{x x \overline{x x} t}+\left(U_{j}^{n+1 / 2}\right)_{\widehat{x}}-\left(U_{j}^{\prime n+1 / 2}\right)_{\widehat{x}} \\
+\kappa\left(U_{j}^{n+1 / 2}, U_{j}^{n+1 / 2}\right)-\kappa\left(U_{j}^{\prime n+1 / 2}, U_{j}^{\prime n+1 / 2}\right)=0, \\
W_{j}^{0}=0 \quad(j=0,1, \ldots, N) .
\end{gathered}
$$

Similarly to the proof of Theorem 2.10, we have

$$
\left\|W^{n}\right\|^{2}+\left\|W_{x}^{n}\right\|^{2}+\left\|W_{x x}^{n}\right\|^{2}=0
$$

This completes the proof of Theorem 2.11.

Remark 2.12. All results above in this paper are correct for initial-boundary value problem of the general Rosenau-RLW equation with finite or infinite boundary.

\section{Numerical Experiments}

In order to test the correction of the numerical analysis in this paper, we consider the following initial-boundary value problems of the general Rosenau-RLW equation:

$$
u_{t}-u_{x x t}+u_{x x x x t}+u_{x}+\left(u^{p}\right)_{x}=0 \quad(0<t<T),
$$




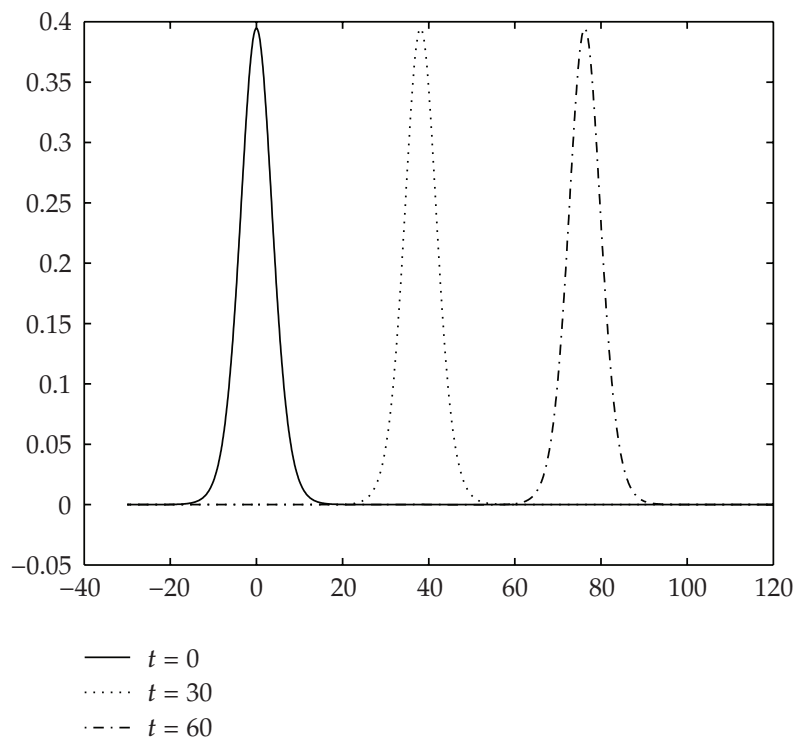

Figure 1: Exact solutions of $u(x, t)$ at $t=0$ and numerical solutions computed by scheme (2.3)-(2.5) at $t=30,60$ for $p=2$.

Table 3: The errors of numerical solutions at $t=60$ with $\tau=h$ for $p=6$.

\begin{tabular}{lcccc}
\hline$h$ & $\left\|u^{n}-U^{n}\right\|$ & $\left\|u^{n}-U^{n}\right\|_{\infty}$ & $\left\|u^{n / 4}-U^{n / 4}\right\| /\left\|u^{n}-U^{n}\right\|$ & $\left\|u^{n / 4}-U^{n / 4}\right\|_{\infty} /\left\|u^{n}-U^{n}\right\|_{\infty}$ \\
\hline 0.4 & $1.787127 \times 10^{-1}$ & $6.353868 \times 10^{-2}$ & & \\
0.2 & $4.598952 \times 10^{-2}$ & $1.649585 \times 10^{-2}$ & 3.885945 & 3.851797 \\
0.1 & $1.156944 \times 10^{-2}$ & $4.159339 \times 10^{-3}$ & 3.975084 & 3.965980 \\
0.05 & $2.892147 \times 10^{-3}$ & $1.040878 \times 10^{-3}$ & 4.000294 & 3.995992 \\
0.025 & $6.585307 \times 10^{-4}$ & $2.375782 \times 10^{-4}$ & 4.391818 & 4.381199 \\
\hline
\end{tabular}

Table 4: Discrete mass $Q^{n}$ and discrete energy $E^{n}$ with $\tau=h=0.1$ at various $t$ for $p=2$.

\begin{tabular}{lcc}
\hline & $Q^{n}$ & $E^{n}$ \\
\hline 10 & 1.89765826296001 & 0.53317523158085 \\
20 & 1.89765826887321 & 0.53317523187251 \\
30 & 1.89765826299393 & 0.53317523117725 \\
40 & 1.89765826556893 & 0.53317523147809 \\
50 & 1.89765826097587 & 0.53317523177618 \\
60 & 1.89765826538488 & 0.53317523107405 \\
\hline
\end{tabular}

Table 5: Discrete mass $Q^{n}$ and discrete energy $E^{n}$ with $\tau=h=0.1$ at various $t$ for $p=3$.

\begin{tabular}{ccc}
\hline & $Q^{n}$ & $E^{n}$ \\
\hline 10 & 2.67260867526530 & 1.11346267885270 \\
20 & 2.67260867623658 & 1.11346267846522 \\
30 & 2.67260867414713 & 1.11346267808394 \\
40 & 2.67260867263988 & 1.11346267871158 \\
50 & 2.67260867287471 & 1.11346267833006 \\
60 & 2.67260867972944 & 1.11346267895871 \\
\hline
\end{tabular}


Table 6: Discrete mass $Q^{n}$ and discrete energy $E^{n}$ with $\tau=h=0.1$ at various $t$ for $p=6$.

\begin{tabular}{lcc}
\hline & $Q^{n}$ & $E^{n}$ \\
\hline 10 & 3.98866332039089 & 1.91761301465671 \\
20 & 3.98866326085426 & 1.91761301473989 \\
30 & 3.98866316768549 & 1.91761301482083 \\
40 & 3.98866319450697 & 1.91761301492744 \\
50 & 3.98866397335989 & 1.91761301400971 \\
60 & 3.98866362197259 & 1.91761301467917 \\
\hline
\end{tabular}

with an initial condition

$$
u(x, 0)=u_{0}(x)
$$

and boundary conditions

$$
u\left(x_{l}, t\right)=u\left(x_{r}, t\right)=0, \quad u_{x x}\left(x_{l}, t\right)=u_{x x}\left(x_{r}, t\right)=0 \quad(0 \leq t \leq T),
$$

where $u_{0}(x)=e^{\ln \left\{(p+3)(3 p+1)(p+1) /\left[2\left(p^{2}+3\right)\left(p^{2}+4 p+7\right)\right]\right\} /(p-1)} \operatorname{sech}^{4 /(p-1)}\left(\left(p-1 / \sqrt{4 p^{2}+8 p+20}\right) x\right)$. Then the exact solution of the initial value problem (3.1)-(3.2) is

$$
u(x, t)=e^{\ln \left\{(p+3)(3 p+1)(p+1) /\left[2\left(p^{2}+3\right)\left(p^{2}+4 p+7\right)\right]\right\} /(p-1)} \operatorname{sech}^{4 /(p-1)}\left[\frac{p-1}{\sqrt{4 p^{2}+8 p+20}}(x-c t)\right]
$$

where $c=\left(p^{4}+4 p^{3}+14 p^{2}+20 p+25\right) /\left(p^{4}+4 p^{3}+10 p^{2}+12 p+21\right)$ is wave velocity.

It follows from (3.4) that the initial-boundary value problem (3.1)-(3.3) is consistent to the boundary value problem (3.3) for $-x_{l} \gg 0, x_{r} \gg 0$. In the following examples, we always choose $x_{l}=-30, x_{r}=120$.

Tables 1,2 , and 3 give the errors in the sense of $L_{2}$-norm and $L_{\infty}$-norm of the numerical solutions under various steps of $\tau$ and $h$ at $t=60$ for $p=2,3$ and 6 . The three tables verify the second-order convergence and good stability of the numerical solutions. Tables 4, 5, and 6 shows the conservative law of discrete mass $Q^{n}$ and discrete energy $E^{n}$ computed by scheme (2.3)-(2.5) for $p=2,3$ and 6 .

Figures 1,2, and 3 plot the exact solutions at $t=0$ and the numerical solutions computed by scheme (2.3)-(2.5) with $\tau=h=0.1$ at $t=30,60$, which also show the accuracy of scheme (2.3)-(2.5).

\section{Acknowledgments}

The authors would like to express their sincere thanks to the referees for their valuable suggestions and comments. This paper is supported by the National Natural Science Foundation of China (nos. 10871117 and 10571110). 


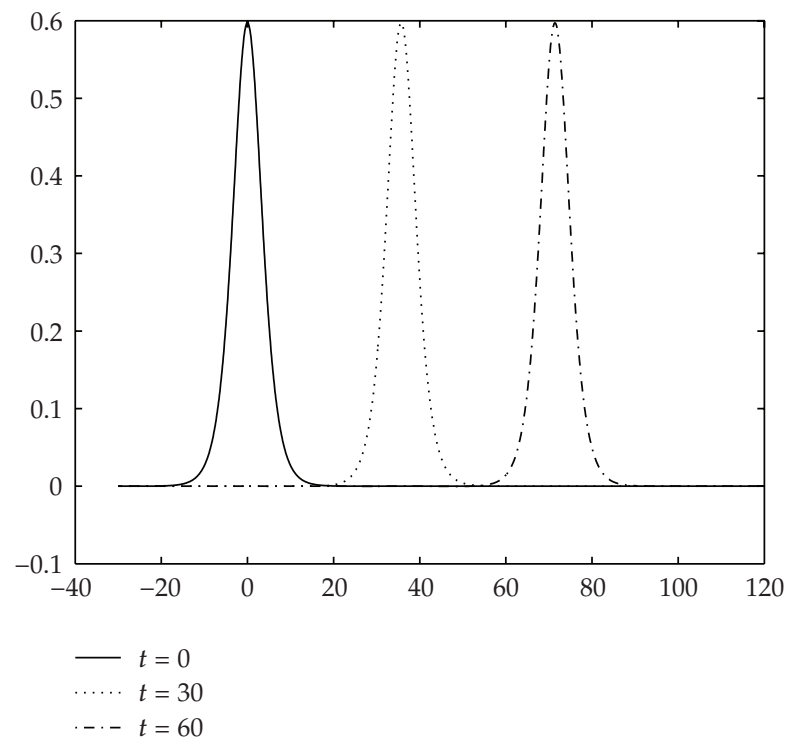

Figure 2: Exact solutions of $u(x, t)$ at $t=0$ and numerical solutions computed by scheme (2.3)-(2.5) at $t=30,60$ for $p=3$.

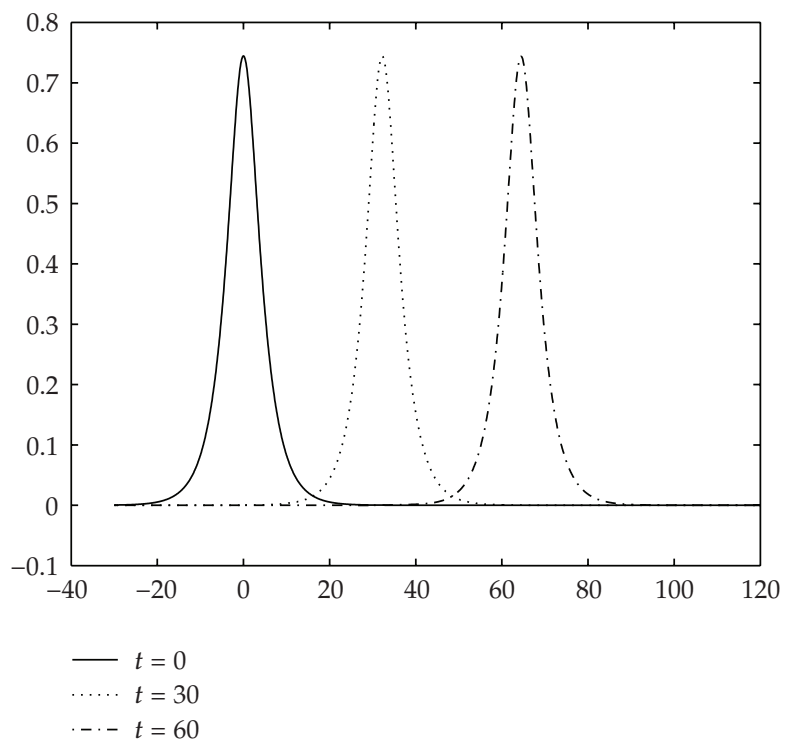

Figure 3: Exact solutions of $u(x, t)$ at $t=0$ and numerical solutions computed by scheme (2.3)-(2.5) at $t=30,60$ for $p=6$.

\section{References}

[1] F. Zhang, V. M. Pérez-García, and L. Vázquez, "Numerical simulation of nonlinear Schrödinger systems: a new conservative scheme," Applied Mathematics and Computation, vol. 71, no. 2-3, pp. 165177, 1995.

[2] S. Li and L. Vu-Quoc, "Finite difference calculus invariant structure of a class of algorithms for the nonlinear Klein-Gordon equation," SIAM Journal on Numerical Analysis, vol. 32, no. 6, pp. 1839-1875, 1995. 
[3] Q. Chang and L. Xu, "A numerical method for a system of generalized nonlinear Schrödinger equations," Journal of Computational Mathematics, vol. 4, no. 3, pp. 191-199, 1986.

[4] Q. Chang, E. Jia, and W. Sun, "Difference schemes for solving the generalized nonlinear Schrödinger equation," Journal of Computational Physics, vol. 148, no. 2, pp. 397-415, 1999.

[5] T.-C. Wang and L.-M. Zhang, "Analysis of some new conservative schemes for nonlinear Schrödinger equation with wave operator," Applied Mathematics and Computation, vol. 182, no. 2, pp. 1780-1794, 2006.

[6] T. Wang, B. Guo, and L. Zhang, "New conservative difference schemes for a coupled nonlinear Schrödinger system," Applied Mathematics and Computation, vol. 217, no. 4, pp. 1604-1619, 2010.

[7] L. Zhang, "A finite difference scheme for generalized regularized long-wave equation," Applied Mathematics and Computation, vol. 168, no. 2, pp. 962-972, 2005.

[8] F. Zhang and L. Vázquez, "Two energy conserving numerical schemes for the sine-Gordon equation," Applied Mathematics and Computation, vol. 45, no. 1, pp. 17-30, 1991.

[9] Y. S. Wong, Q. Chang, and L. Gong, "An initial-boundary value problem of a nonlinear Klein-Gordon equation," Applied Mathematics and Computation, vol. 84, no. 1, pp. 77-93, 1997.

[10] Q. S. Chang, B. L. Guo, and H. Jiang, "Finite difference method for generalized Zakharov equations," Mathematics of Computation, vol. 64, no. 210, pp. 537-553, 1995.

[11] J. S. Hu and K. L. Zheng, "Two conservative difference schemes for the generalized Rosenau equation," Boundary Value Problems, vol. 2010, Article ID 543503, 18 pages, 2010.

[12] B. $\mathrm{Hu}, \mathrm{Y} . \mathrm{Xu}$, and J. Hu, "Crank-Nicolson finite difference scheme for the Rosenau-Burgers equation," Applied Mathematics and Computation, vol. 204, no. 1, pp. 311-316, 2008.

[13] F. E. Browder, "Existence and uniqueness theorems for solutions of nonlinear boundary value problems," in Proceedings of Symposia in Applied Mathematics, vol. 17, pp. 24-49, American Mathematical Society, Providence, RI, USA, 1965.

[14] Y. Zhou, Applications of Discrete Functional Analysis to the Finite Difference Method, International Academic Publishers, Beijing, China, 1991. 